\title{
Epidemiologie okulärer Manifestationen bei Autoimmunkrankheiten
}

\author{
Katie Glover Deepakkumar Mishra Thakur Raghu Raj Singh \\ School of Pharmacy, Medical Biology Centre, Queen's University Belfast, Belfast, Vereinigtes Königreich
}

\author{
Schlüsselwörter \\ okuläre Manifestationen · Autoimmunerkrankungen . \\ Retinopathie · Epidemiologie · systemische \\ Autoimmunerkrankungen
}

\begin{abstract}
Zusammenfassung
Die weltweite Prävalenz von Autoimmunkrankheiten nimmt zu. Infolgedessen haben auch die mit Autoimmunkrankheiten verbundenen okulären Komplikationen zugenommen, die von leichten Symptomen bis hin zur Bedrohung des Sehvermögens reichen. Diese okulären Manifestationen können durch die Krankheit selbst
\end{abstract}

oder durch die Behandlung zur Bekämpfung der primären Autoimmunerkrankung verursacht werden. Diese Übersicht gibt einen detaillierten Einblick in die epidemiologischen Faktoren, die die zunehmende Prävalenz von Augenkomplikationen im Zusammenhang mit verschiedenen Autoimmunerkrankungen beeinflussen.

(c) 2021 Glover, Mishra and Singh

\section{Einleitung}

Autoimmunkrankheiten entstehen dadurch, dass das körpereigene Immunsystem Selbstantigene angreift [1], und werden je nach Zielgebiet im Körper entweder als organspezifisch oder systemisch eingestuft. Obwohl über 80 Autoimmunkrankheiten bekannt sind, ist die genaue Ätiologie vieler dieser Krankheiten noch unbekannt. Genetische und umweltbedingte Faktoren sind ausschlaggebend für die Anfälligkeit für und die Prävalenz und den Schweregrad von Krankheiten bedingt durch das zelluläre Immunsystem.

Die vielfältigen okulären Manifestationen, die mit verschiedenen Autoimmunerkrankungen in Verbindung stehen, werden häufig übersehen und in ihrer Bedeutung unterschätzt. Diese Manifestationen reichen von leichten Störungen bis hin zu bedrohlichen $\mathrm{Zu}$ ständen, die ein sofortiges medizinisches Eingreifen erfordern. Das Auge ist ein empfindliches Organ mit einer Mikroumgebung, die empfindlich auf systemische Veränderungen im Körper reagiert und auch als erster Indikator für eine zugrunde liegende Autoimmunerkrankung dienen kann [2, 3]. Augensymptome können auch während der aktiven Krankheit oder Jahre nach der Diagnose auftreten. Eine Verzögerung der Behandlung dieser Krankheitserscheinungen hat unmittelbare Auswirkungen auf die Lebensqualität der Patienten, und in einigen Fällen besteht zweifellos die Gefahr einer Sehbehinderung.

Jeder Teil des Auges ist ein potenzielles Ziel für autoimmunbedingte Komplikationen. Idealerweise sollte eine Augenuntersuchung ein Routinebestandteil des Krankheitsmanagements werden, um auftretende Augensymptome rechtzeitig zu diagnostizieren, zu untersuchen und zu behandeln. Darüber hinaus sollte die Bedeutung regelmäßiger Screenings, auch bei asymptomatischen Patienten, aufgrund des Potenzials für akute, sehkraftbedrohende Augenkomplikationen, die bei mehreren der in dieser Übersicht behandelten Autoimmunkrankheiten beobachtet werden, hervorgehoben werden.

Autoimmunerkrankungen nehmen weltweit zu, von einer geschätzten Prävalenz von 3,2\% zwischen 1965 und 1995 auf 19,1 \pm 43,1\% im Jahr 2018 [4, 5]. Bis 2026 wird der weltweite Markt für die Diagnose von Autoimmunkrankheiten, der derzeit einen Wert von 4,1 Mrd. USD hat, schätzungsweise 6,3 Mrd. USD er- 
Abb. 1. Klassifikation von Autoimmunkrankheiten.

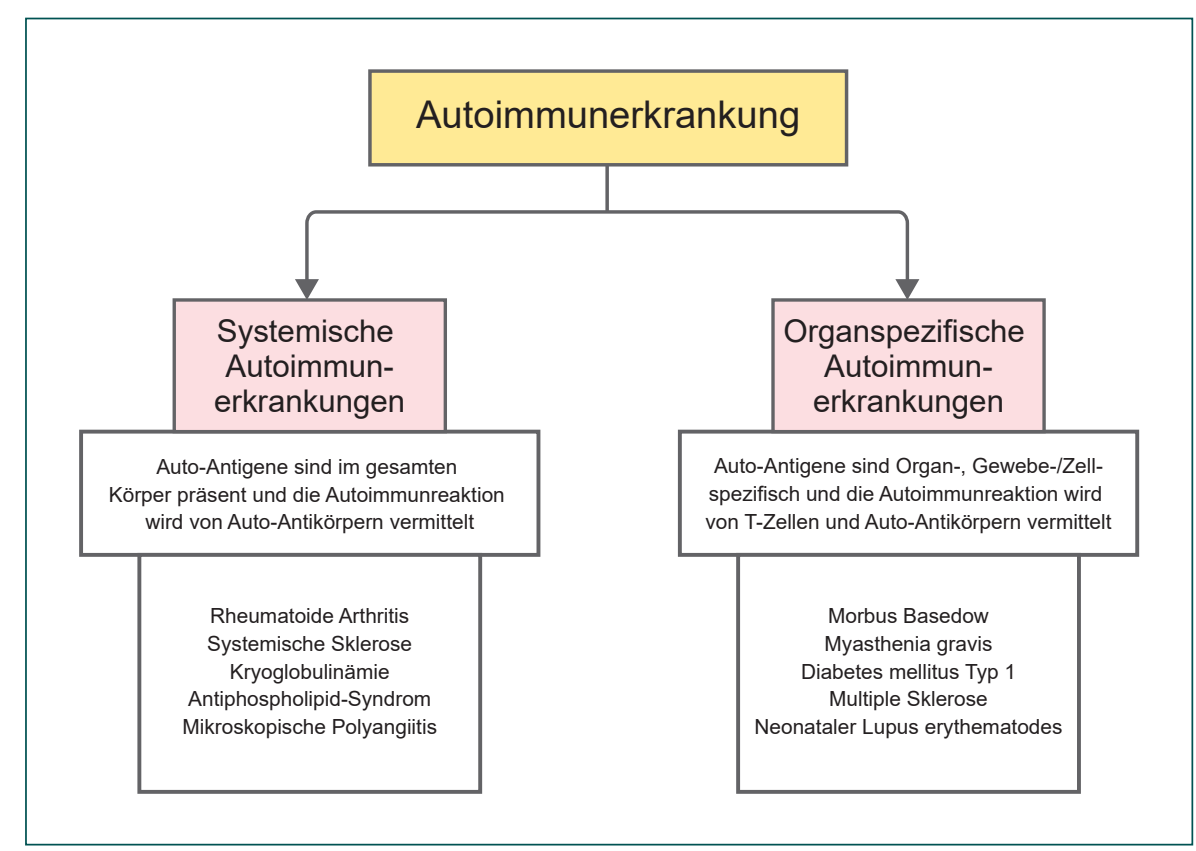

Abb. 2. Verschiedene Körperstellen, die von Autoimmunerkrankungen betroffen sein können.

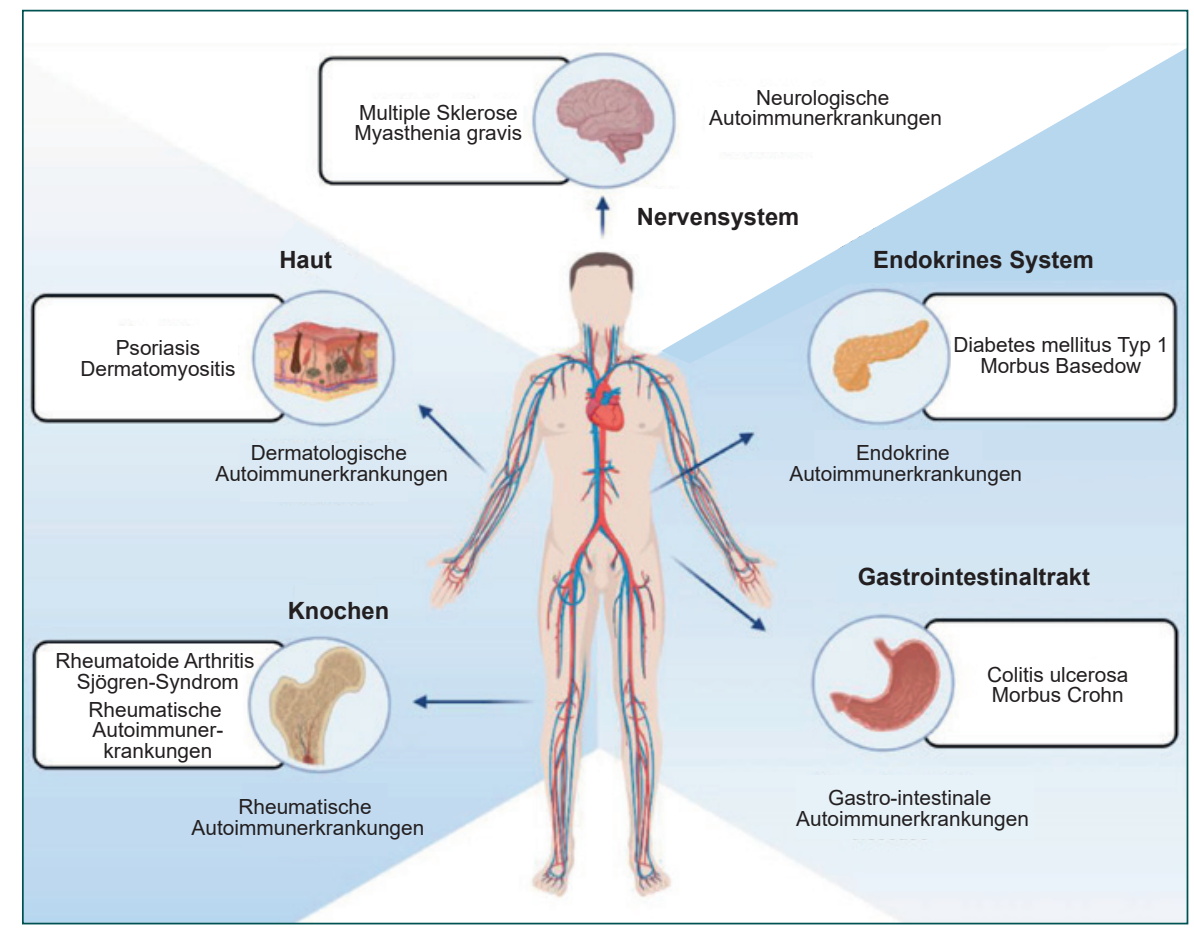

reichen [6]. Einige Gründe für diesen Anstieg sind auf die genetische Veranlagung in einer alternden Bevölkerung und auf verbesserte Diagnosetechniken zurückzuführen. Die zunehmende Prävalenz wurde jedoch stärker von Umweltfaktoren beeinflusst, was auf eine Möglichkeit schließen lässt, wie diese Probleme verringert werden können.

Darüber hinaus ist Polyautoimmunität oder ein multiples Autoimmun-Syndrom bei einem einzigen Patienten keine Seltenheit, wie z.B. die Verbindung zwischen rheumatoider Arthritis, Schilddrüsenentzündung und Diabetes mellitus Typ 1 [7]. Solche
Bedingungen erhöhen das Risiko systemischer Manifestationen, einschließlich solcher, die das Auge betreffen.

Angesichts der zunehmenden Prävalenz von Autoimmunkrankheiten in der Weltbevölkerung, des zusätzlichen Risikos einer Polyautoimmunität und der alternden Bevölkerung können wir nur vermuten, dass die begleitenden okulären Manifestationen dieser Krankheiten ebenfalls zunehmen werden. Es wurden verschiedene Übersichtsarbeiten veröffentlicht, um die Prävalenz von okulären Manifestationen verschiedener Autoimmunkrankheiten hervorzuheben. Eine Übersichtsarbeit, die die epidemiologische 


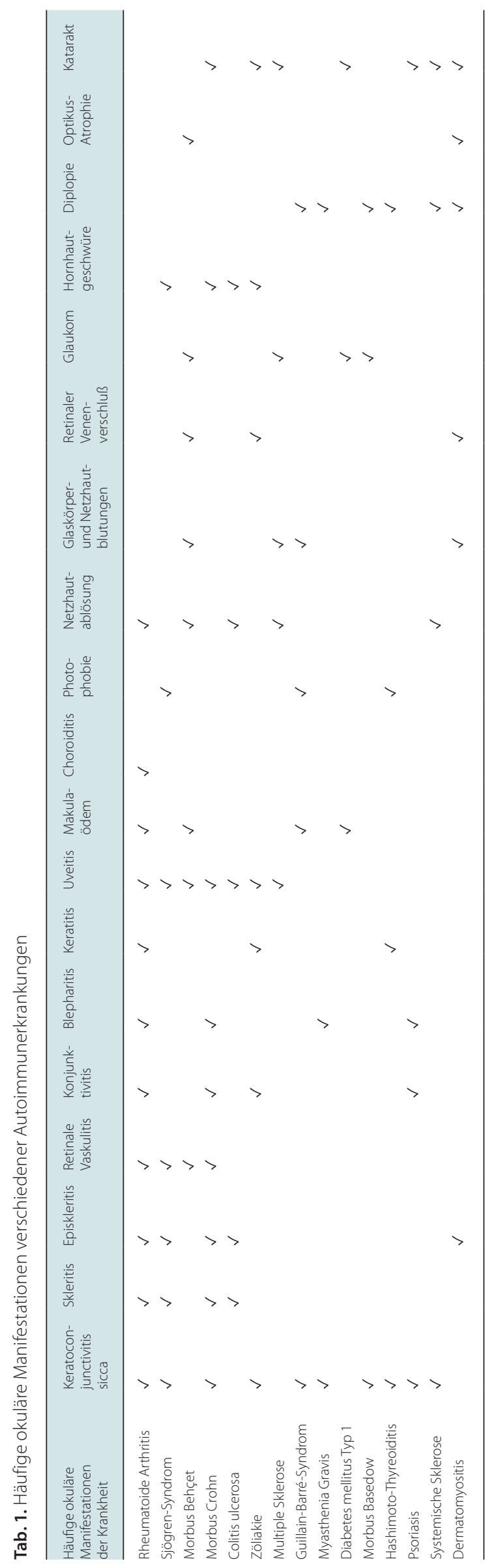

Prävalenz zusammen mit aktueller Literatur hervorhebt, ist jedoch sehr wünschenswert [8-11]. Diese Übersichtsarbeit soll das Bewusstsein für die verschiedenen Augenkomplikationen im Zusammenhang mit Autoimmunkrankheiten und für die am stärksten gefährdeten Personen schärfen, damit beim Screening und bei der Diagnose mit besonderer Sorgfalt vorgegangen werden kann.

\section{Autoimmunerkrankungen und ihre Auswirkungen auf das Auge}

Autoimmunerkrankungen lassen sich grob in systemische und organspezifische Erkrankungen einteilen (Abb. 1). Bei systemischen Autoimmunerkrankungen richtet sich die Autoimmunität ausschließlich gegen das ubiquitär (universell) exprimierte Selbstantigen und führt zu Antikörper-vermittelten Endorganschäden. Autoimmunerkrankungen betreffen auch verschiedene Teile des Körpers, einschließlich der Haut und des Magen-Darm-Systems (Abb. 2). Autoantikörper spielen im Vergleich zu T-Zellen eine bedeutende Rolle bei systemischen Autoimmunerkrankungen. Bei organspezifischen Autoimmunerkrankungen zielen die Autoantikörper und die T-Zellen auf die organ-/gewebe-/zellspezifischen Antigene ab, was zu einer spezifischen und zielgerichteten Autoimmunreaktion führt.

Augenkomplikationen infolge von Autoimmunität reichen von weniger schwerwiegenden Auswirkungen, z.B. topische Konjunktivitis und Skleritis, bis hin zu chronischen und schwerwiegenden Auswirkungen, z.B. Neuropathien und choroidale Angiogenese. Wie in Tabelle 1 zusammengefasst, ist ein breites Spektrum von Autoimmunerkrankungen häufig mit gemeinsamen okulären Manifestationen verbunden; einige okuläre Manifestationen sind jedoch krankheitsspezifisch (Tab. 2). Verschiedene Autoimmunerkrankungen, die in dieser Übersicht besprochen werden, stehen in Zusammenhang mit nachteiligen okulären Manifestationen; weitere Einzelheiten über die Prävalenz und die Symptome der okulären Auswirkungen mehrerer Klassen von Autoimmunerkrankungen werden ebenfalls diskutiert.

\section{Rheumatische Autoimmunerkrankungen}

Autoimmunrheumatische Erkrankungen (ARD) sind eine vielfältige Gruppe von Erkrankungen, die in erster Linie Gelenke, Knochen, Muskeln und Bindegewebe betreffen, wobei die rheumatoide Arthritis die häufigste ist.

\section{Rheumatoide Arthritis}

Rheumatoide Arthritis ist eine chronische Erkrankung, die eine systemische Polyarthritis verursacht, die im Allgemeinen in bilateraler Form vorliegt und durch eine Entzündung des Synovialgewebes gekennzeichnet ist. In den letzten Jahren lagen die Schätzungen der globalen Prävalenz zwischen $1 \%$ und $2 \%$ [12, 13].

Die Keratokonjunktivitis sicca oder das trockene Auge ist die häufigste okuläre Präsentation der rheumatoiden Arthritis und tritt bei $10-35 \%$ der Patienten auf. Weitere häufige Manifestationen sind Episkleritis, Skleritis, periphere ulzerative Keratitis (PUK) und retinale Vaskulitis. In einer neueren Studie wies ein Drittel 
Tab. 2. Seltene okuläre Manifestationen verschiedener Autoimmunerkrankungen

\begin{tabular}{|c|c|}
\hline Autoimmunkrankheit & Ungewöhnliche okuläre Manifestationen im Zusammenhang mit Autoimmunkrankheiten \\
\hline Rheumatoide Arthritis & periphere Ulzeration, Pterygium, Ausdünnung des peripheren Stromas, akute zentrale Hornhautschmelze, Choroiditis \\
\hline Sjögren-Syndrom & $\begin{array}{l}\text { Netzhautvaskulitis, Einschmelzen der Hornhaut, Hornhautperforation, konjunktivale epitheliale Verhornung, } \\
\text { Optikusneuropathie, steriles Hornhautgeschwür }\end{array}$ \\
\hline Morbus Behçet & $\begin{array}{l}\text { Hypopyon, Papillitis, Chorioretinitis, retinale Periphlebitis, Periarteritis, posteriore Synechien, Irisbombe, keratische } \\
\text { Präzipitate, epiretinale Membran }\end{array}$ \\
\hline Morbus Crohn & $\begin{array}{l}\text { subepitheliale Infiltrate, perivaskuläre Umhüllung, Lidschwellung, Lidrand, orbitale Myositis, Sehnervenentzündung, } \\
\text { Dyakroadenitis, Ptosis palpebralis, choroidale Neovaskularisation, zentrale seröse Chorioretinopathie }\end{array}$ \\
\hline Ulzerative Colitis & $\begin{array}{l}\text { Orbitaschwellung, Vaskulitis, Iritis, zentrale seröse Chorioretinopathie, Keratopathie, Aderhauterguss, choroidale } \\
\text { Neovaskularisation, Hirnnervenlähmung, Sehnervenentzündung }\end{array}$ \\
\hline Zöliakie & $\begin{array}{l}\text { orbitale Myositis, Keratomalazie, mikrobielle Keratitis, Retinopathie, Pseudotumor cerebri, Nyktalopie, Schilddrüsen- } \\
\text { assoziierte Orbitopathie }\end{array}$ \\
\hline Multiple Sklerose & $\begin{array}{l}\text { Hirnnervenlähmungen, Sehnervenentzündung, Optikusneuritis, internukleäre Ophthalmoplegie, Nystagmus, Pars } \\
\text { planitis, retinale Periphlebitis, Oszillopsie, verminderte Farbwahrnehmung, Läsionen des Chiasmas }\end{array}$ \\
\hline Guillain-Barré-Syndrom & $\begin{array}{l}\text { Akkommodationsinsuffizienz, Papillophlebitis, Watteflecken, Pupillendysfunktion, Ophthalmoparese, Esotropie, } \\
\text { Lagophthalmus, Ektropium, Hornhautempfindlichkeit, Papillenödem, Colgan-Lidzucken, Mydriasis, vertikale Blickparese }\end{array}$ \\
\hline Myasthenia gravis & $\begin{array}{l}\text { internukleäre Ophthalmoplegie, Ptosis, Schilddrüsenerkrankungen, Ophthalmoparese, Lagophthalmus, } \\
\text { Orbicularisschwäche }\end{array}$ \\
\hline Diabetes mellitus Typ 1 & diabetische Retinopathie, Akkommodationsinsuffizienz, reduzierte Gefäßdichte, Hornhauterosion, Hornhauthypoästhesie \\
\hline Morbus Basedow & $\begin{array}{l}\text { Schilddrüsen-assoziierte Ophthalmologie, Hornhauthysterese, Proptose, Meibom-Drüsen-Dysfunktion, Augenläsionen, } \\
\text { Bindehauterythem, Augenlidödem }\end{array}$ \\
\hline Hashimoto-Thyreoiditis & $\begin{array}{l}\text { Schilddrüsen-assoziierte Ophthalmologie, Hornhauthysterese, Ptosis, reduzierte Farbwahrnehmung, Retraktion des } \\
\text { oberen Augenlids, Chemosis, Vorfall der Konjunktiva, Proptosis, Exophthalmos, von Graefe'sches Zeichen }\end{array}$ \\
\hline Psoriasis & $\begin{array}{l}\text { Psoriasis der Augenlider, konjunktivale Läsionen, Xerosis, Ektropium, orbitale Myositis, Hornhautpigmentdispersion, } \\
\text { Hornhauttrübungen, Ptosis, peripheres Hornhautschmelzsyndrom, Funktionsstörung der Meibomschen Drüsen }\end{array}$ \\
\hline Systemische Sklerose & $\begin{array}{l}\text { Telangiektasie, Keratokonus, Iris-Transillumination, Funktionsstörung der Meibomschen Drüsen, Keratopathie, } \\
\text { Hornhautastigmatismus }\end{array}$ \\
\hline Dermatomyositis & $\begin{array}{l}\text { Ptosis, Strabismus, Bindehautödem, Nystagmus, Iritis, Watteflecken, internukleäre Ophthalmoplegie, Papillenödem, } \\
\text { orbitale Myositis, canthale Narben }\end{array}$ \\
\hline
\end{tabular}

der Patienten mit rheumatoider Arthritis okuläre Manifestationen auf, wobei die Keratokonjunktivitis sicca $85 \%$ der okulären Erkrankungen ausmachte [14]. Das trockene Auge ist auf eine Schädigung der Tränendrüse durch angreifende B- und T-Lymphozyten zurückzuführen. Obwohl es derzeit keine wirksame Behandlung gibt, können Augentropfen zur Linderung der Symptome Juckreiz, Rötung und Fremdkörpergefühl eingesetzt werden. In schwereren Fällen kann ein Verschluss der ableitenden Tränenpünktchen oder eine Tarsorrhaphie erforderlich sein. Bei Patienten mit rheumatoider Arthritis wurde kein Zusammenhang zwischen dem Schweregrad der Erkrankung und dem Schweregrad des trockenen Auges festgestellt. Ein eher schwerere Keratokonjunktivitis sicca war mit einer längeren Krankheitsdauer verbunden [15]. Es wurde festgestellt, dass die Hornhaut von Patienten mit rheumatoider Arthritis signifikant dünner ist als bei gesunden Kontrollkohorten, wobei die Verringerung der Hornhautdicke mit zunehmender Hornhautkrümmung abnahm [16].

Eine Episkleritis tritt bei bis zu 10\% der Patienten mit rheumatoider Arthritis auf und wird durch eine Entzündung der suprachoroidalen Schicht verursacht. Zur Verengung der Blutgefäße können topische Augentropfen verabreicht werden, die jedoch nicht auf die tief in der Sklera liegenden Gefäße wirken, was eine wirksame Behandlung erschwert. Bei Patienten mit rheumatoider Arthritis wurden im Vergleich zu gesunden Kontrollgruppen dünnere Aderhautschichten und ein erhöhter Widerstand gegen den Blutfluss festgestellt [17]. Dabei wurde jedoch kein Zusammenhang mit dem Schweregrad der Erkrankung festgestellt, was auf das Risiko potenziell schwerer okulärer Manifestationen bei gut behandelten Patienten mit rheumatoider Arthritis hinweist.

Skleritis macht 10\% der okulären Komplikationen bei Patienten mit rheumatoider Arthritis aus [12], kann jedoch erheblich schmerzhafter sein. PUK ist bei rheumatoider Arthritis selten und wird hauptsächlich in Fallstudien berichtet. Ein Fall zeigt jedoch die rasche Entwicklung von allgemeinen Symptomen zu einer schweren bilateralen PUK mit Sehverlust [18]. Skleritis und PUK können sich zu einer retinalen Vaskulitis entwickeln [19], die zum Verlust des Sehvermögens führen kann, wenn nicht rechtzeitig eingegriffen wird. In einigen Fällen verläuft die retinale Vaskulitis asymptomatisch, sodass ein regelmäßiges Screening aller Patienten für die Erkennung und Behandlung von okulären Manifestationen von größter Bedeutung ist. 
Abb. 3. (a) Beobachtung der limbalen Plattenepithel-Neoplasie der Augenoberfläche zusammen mit Keratin-Plaques bei Patienten mit Morbus Crohn. (b) Histopathologie des invasiven Plattenepithelkarzinoms nach Exzision des Tumors unter Verwendung von Hämatoxylin-Eosin (H\&E)-Färbung. Bild verwendet mit Genehmigung von Triphathy et al. [35].
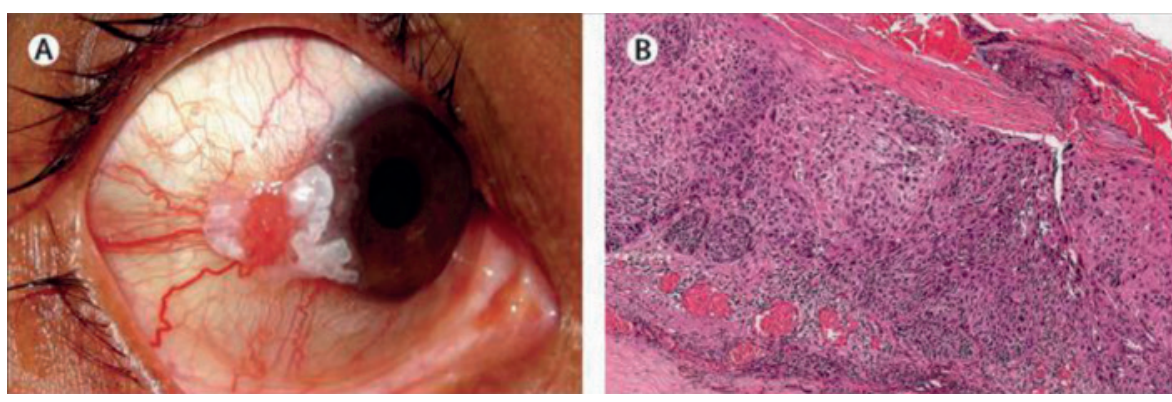

\section{Sjögren-Syndrom}

Das Sjögren-Syndrom ist eine chronische Entzündungskrankheit, die insbesondere die Tränen- und Speicheldrüsen betrifft. Patienten mit Sjögren-Syndrom zeigen Sicca-Symptome aufgrund einer entzündlichen Schädigung der exokrinen Tränen- und Speicheldrüsen. Die aktuelle weltweite Prävalenz wird auf bis zu $1 \%$ der Bevölkerung geschätzt [20].

Einer von drei Patienten mit Sjögren-Syndrom weist okuläre Symptome auf, von denen 13\% das Sehvermögen bedrohen [21]. Wie bei der rheumatoiden Arthritis ist die Keratokonjunktivitis sicca die häufigste okuläre Manifestation. Als Vorstadium weist 1 von 10 amerikanischen Patienten über 50 Jahren, bei denen ein trockenes Auge diagnostiziert wird, letztlich ein Sjögren-Syndrom auf [22]. Unglücklicherweise liegen bei denjenigen, bei denen die Diagnose gestellt wird, schätzungsweise 10 Jahre zwischen dem ersten Auftreten von Augensymptomen und der Diagnose des Sjögren-Syndroms [21], was darauf hindeutet, dass die Bedeutung der okulären Manifestationen des Sjögren-Syndroms nicht ausreichend bekannt ist.

Andere Erkrankungen wie Episkleritis, Skleritis, retinale Vaskulitis und eine Einschmelzung oder Perforation der Hornhaut werden häufig mit dem primären Sjögren-Syndrom in Verbindung gebracht, wobei einige Untersuchungen darauf hindeuten, dass Männer ein höheres Risiko haben, diese okulären Symptome zu entwickeln [23], obwohl diese Feststellung durchaus infrage gestellt werden kann [24].

\section{Morbus Behçet}

Morbus Behçet ist gekennzeichnet durch eine chronische Vaskulitis mehrerer Organe, einschließlich der Augen und des Nervensystems. Morbus Behçet weist eine hohe Inzidenz von okulären Manifestationen auf, wobei bei $70 \%$ der Patienten irgendeine Form der Augenerkrankung festgestellt wird [25]. Bei Morbus Behçet sind die Augensymptome in der Regel nicht die ersten Symptome, die sich bemerkbar machen, ihre Bedeutung darf jedoch nicht unterschätzt werden.

Eine rezidivierende bilaterale Uveitis tritt bei etwa zwei Dritteln der Patienten mit Morbus Behçet auf [26], wobei bei 25\% dieser Patienten eine Erblindung eintreten kann [27]. Darüber hinaus können bereits in einem frühen Stadium der Behçet-assoziierten Uveitis schwerwiegende Komplikationen auftreten, wie Netzhautläsionen (75-80\%) [28], Katarakte (39,5\%) und Sekundärglaukom (17,1\%) [29], die zu einer Sehbehinderung führen.
Bei einem männlichen Patienten, der neben einem Makulaödem und einem Bandscheibenödem eine verminderte Sehschärfe und intraretinale Blutungen aufwies, wurde ein Versuch mit systemischen Kortikosteroiden, Immunsuppressiva und einem intravitrealen Dexamethason-Implantat durchgeführt. Obwohl sich das Makulaödem zurückbildete, blieb eine verminderte Sehschärfe zurück, was die irreversiblen Auswirkungen der Behçet-assoziierten Uveitis verdeutlicht [25]. In einer Studie trat bei über der Hälfte der Patienten ein Makulaödem auf. Am häufigsten trat jedoch eine retinale Periphlebitis auf [29].

\section{Magen-Darm-Autoimmunerkrankungen}

Bei gastrointestinalen Autoimmunkrankheiten richtet sich das Immunsystem gegen die Organe des Magen-Darm-Trakts. Der derzeitige Mangel an Bewusstsein für diese Krankheiten wird anhand von Fallstudien in der Literatur nachgewiesen. So ist beispielsweise nur wenig erforscht, wie die Hornhaut bei Patienten mit chronisch-entzündlichen Darmerkrankungen (CED) beeinträchtigt wird. Neuere Untersuchungen haben jedoch ergeben, dass Morbus-Crohn-Patienten, die keine Symptome einer Augenbeteiligung aufwiesen, eine verringerte Hornhautdicke und eine geringere Tränenmenge aufwiesen [30].

\section{Morbus Crohn}

Morbus Crohn ist durch Entzündungsschübe im Magen-DarmTrakt gekennzeichnet, die zu Narbenbildung und Geschwüren führen. Morbus Crohn kann jede Altersgruppe betreffen, tritt jedoch am häufigsten im späten Teenageralter auf. Neuere Studien deuten auf einen Anstieg der Prävalenz hin [31].

Bis zu 12\% der Morbus-Crohn-Patienten weisen okuläre Manifestationen auf, wobei die Episkleritis die häufigste ist, dicht gefolgt von Skleritis und Uveitis [32]. Eine posteriore Uveitis bei Morbus Crohn wird mit einer gemeldeten Inzidenz von 5,6 Personen pro 100000 Einwohnern gemeldet [33]. Oral eingenommenes Prednisolon linderte bei 1 Patienten Augenläsionen, eine leichte Vitritis und intraretinale Blutungen, die vor der Diagnose des Morbus Crohn aufgetreten sind, ohne dass über bleibende Augenschäden berichtet wurde [34]. Okuläre Neoplasien wurden auch mit Morbus Crohn in Verbindung gebracht (Abb. 3) [35].

Okuläre Manifestationen treten vor allem in den frühen Stadien der Krankheit auf [36], können aber auch während der aktiven Krankheit oder in der Remission auftreten. So wurde beispielsweise eine orbitale Myositis, die bei Morbus Crohn zwar immer 
noch selten ist, häufiger als bei Colitis ulcerosa gemeldet. Bei einem Patienten kam es zu einem akuten Ausbruch einer orbitalen Myositis während einer Phase der Krankheitsremission, die mit Prednisolon und Adalimumab schnell abklang [37].

$\mathrm{Zu}$ den weniger häufigen Manifestationen gehören «Hornhautgeschwüre, Blepharitis, Katarakte, Konjunktivitis, Makulahämorrhagie, subepitheliale Infiltrate, perivaskuläre Umhüllungen und retinale Vaskulitis» [36]. Das trockene Auge tritt bei Morbus Crohn im Vergleich zur Colitis ulcerosa häufiger auf [30, 38]. Die retinale Vaskulitis wurde 5 Jahre vor den gastrointestinalen Symptomen entdeckt, die eine Diagnose des Morbus Crohn ermöglichten [2].

\section{Colitis ulcerosa}

Colitis ulcerosa (CU) ist eine andere Form von CED, die nur den Dickdarm und das Rektum betrifft. Trotz der zunehmenden Prävalenz bleibt die genaue Ätiologie der CU unbekannt.

Auch bei Patienten mit Colitis ulcerosa wird eine Inzidenz von okulären Manifestationen zwischen 4-12\% berichtet, wobei Uveitis und Iritis häufiger mit Colitis ulcerosa als mit Morbus Crohn in Verbindung gebracht werden [32]. Eine Studie ergab, dass 83\% der Patienten okuläre Manifestationen aufwiesen, wobei Katarakte und Konjunktivitis am häufigsten waren [38]. Es liegen widersprüchliche Daten vor, wobei einige Studien über ein höheres Ausmaß okulärer Manifestationen bei Colitis ulcerosa berichteten $[39,40]$, und eine Studie berichtete, dass Patienten mit Morbus Crohn ein höheres Risiko für die Entwicklung okulärer Manifestationen haben [41].

Weitere seltene Manifestationen sind der Zentralvenenverschluss der Netzhaut, Vaskulitis, periphere Hornhautgeschwüre, Hornhautinfiltrate, zentrale seröse Chorioretinopathie und Netzhautablösung. Keratopathie und Nachtblindheit infolge von VitaminA-Mangel können auch auftreten [42]. Zu den weniger häufig auftretenden okulären Nebenwirkungen gehören Vitritis, Aderhauterguss, choroidale Neovaskularisation, Hirnnervenlähmung und Sehnervenentzündung. Obwohl sie bei Morbus Crohn häufiger vorkommt, gibt es Fallberichte über eine orbitale Myositis im Zusammenhang mit Colitis ulcerosa [43, 44]. Darüber hinaus wurde über Fälle von Colitis ulcerosa-Patienten mit ZVV (Zentralvenenverschluss im Auge)) berichtet, die in einigen Fällen zu einem dauerhaften Sehverlust führten [2, 45]. Eine orbitale Schwellung wurde bei einem Patienten mit Colitis ulcerosa berichtet, was den ersten bekannten Zusammenhang zwischen Colitis ulcerosa und IgG4-ROD (IgG4-related ophthalmic disease) darstellt [46].

\section{Zöliakie}

Zöliakie ist eine chronische Erkrankung des Dünndarms, von der 1-2\% der Weltbevölkerung betroffen sind [47] und die durch den langfristigen Verzehr von Gluten ausgelöst wird. Außerdem haben bis zu 15\% der Zöliakiepatienten das Sjögren-Syndrom als sekundäre Autoimmunerkrankung [48], was das Risiko von Augenproblemen erhöht.

Ein Vitamin-D-Mangel tritt bei 20-60\% der Zöliakiepatienten auf [47], was zu einer möglichen Kataraktbildung führen kann.
Außerdem haben Zöliakiepatienten im Vergleich zur Allgemeinbevölkerung aufgrund von Krankheitsfaktoren wie oxidativem Stress und Dehydrierung durch chronischen Durchfall ein höheres Risiko, an Katarakten zu erkranken. Auch ein Vitamin-AMangel kann bei bis zu einem Drittel der Patienten auftreten und zu Nyktalopie, trockenen Augen und Hornhautgeschwüren führen [49].

Augenmanifestationen können auf eine asymptomatische Zöliakie hinweisen [50], einschließlich filamentärer Keratitis, Keratomalazie, mikrobieller Keratitis, Hornhautgeschwüren und Diplopie. Eine Diplopie tritt häufiger bei weiblichen Patienten auf, die dann auf eine orbitale Myositis untersucht werden sollten. Reduzierte Funktionen des Tränenfilms und Anomalien in den Strukturen des Hornhautepithels treten bei Zöliakiepatienten ebenfalls auf [51]. Retinopathie wurde mit Zöliakie in Verbindung gebracht, insbesondere in Verbindung mit Diabetes mellitus Typ 1 [52]. Zöliakie wurde mit anteriorer Skleritis in Verbindung gebracht [53], bei der okuläre und gastrointestinale Symptome nach dem Verzehr von Gluten zusammentrafen, was sich nach der Aufnahme einer glutenfreien Ernährung besserte.

Die meisten Fälle von Zöliakie bleiben unentdeckt, weil sie asymptomatisch sind und das Bewusstsein für die Krankheit fehlt, was verheerende Folgen für das Auge haben kann. Dogan et al. beobachteten dünnere subfoveale Aderhautschichten bei Zöliakiepatienten, bei denen die Diagnose erst nach mehr als 60 Monaten gestellt wurde, was auf die Notwendigkeit einer effizienteren Diagnose und eines besseren Bewusstseins hinweist, um die Entstehung von Augenkomplikationen zu verhindern [54].

\section{Neurologische Autoimmunerkrankungen}

Neurologische Autoimmunerkrankungen wie multiple Sklerose sind das Ergebnis eines immunologisch bedingten Entzündungsangriffs auf das zentrale Nervensystem, der zu schweren Behinderungen und systemischen Symptomen führt.

\section{Multiple Sklerose}

Multiple Sklerose (MS) ist eine fortschreitende Erkrankung des zentralen Nervensystems, die zu schweren Behinderungen führt. Die Autoimmunreaktion führt zu einer Demyelinisierung von Nervenfasern, was weiter zu einer verminderten oder unterbundenen Signalübertragung entlang der Nerven führt. Studien deuten darauf hin, dass die Augenbewegung in 70\% der MS-Fälle beeinträchtigt ist [55]. Die Prävalenz von multipler Sklerose nimmt mit 2 Millionen Fällen weltweit zu [56].

Eine Optikusneuritis ist eine häufige Manifestation aufgrund von Läsionen des Sehnervs und betrifft 7 von 10 MS-Patienten [57], insbesondere bei Frauen. Die Sehnervenentzündung ist in der Regel durch akuten Sehverlust und Augenschmerzen gekennzeichnet; es wird jedoch auch von schweren Komplikationen berichtet. Diese Komplikationen, die auch als atypische Sehnervenentzündung bezeichnet werden, umfassen Netzhautblutungen, Optikusatrophie und einen geschwollenen Sehnerv und erfordern eine intensive Behandlung, um einen Sehverlust oder eine chronische Sehnervenentzündung zu verhindern. 
Über Pupillenstörungen wurde auch bei MS berichtet, mit einer ungefähren Prävalenz von 60\% der MS-Patientenkohorten [58]. Darüber hinaus tritt bei $30 \%$ der MS-Fälle eine internukleäre Ophthalmoplegie auf, die durch Läsionen des Fasciculus longitudinalis medialis zu einer eingeschränkten Augenbewegung führt [59]. Nystagmus ist auch bei Patienten mit multipler Sklerose häufig, wobei der erworbene Pendelnystagmus (APN) am häufigsten auftritt [60]. APN kann zu schweren Sehstörungen führen, die jedoch bei rechtzeitiger und angemessener Behandlung rückgängig gemacht werden können [61].

Die Häufigkeit der MS-assoziierten Uveitis variiert in der Literatur, wobei die höchste bekannte Vorhersage bei 36\% liegt [62]. Wenn sie übersehen wird, kann eine MS-assoziierte Uveitis zu Katarakten, Glaukomen, zystoiden Makulaödemen oder Netzhautablösungen führen.

\section{Guillain-Barré-Syndrom}

Mit einer geschätzten Inzidenz von 1 von 100000 [63] verursacht das Guillain-Barré-Syndrom Demyelinisierung und axonale Degeneration, typischerweise nach einer vorangegangenen Infektion des Magen-Darm-Trakts oder der Atemwege. Trotz verschiedener Subtypen liegen dem Guillain-Barré-Syndrom eine Verschlechterung der Nervenleitung sowie eine Schwäche der Gliedmaßen und der Gesichtsmuskulatur zugrunde. Die Symptome schreiten schnell voran und erreichen oft innerhalb weniger Wochen den Höhepunkt der Krankheit.

Akkommodationsinsuffizienz und Ptosis können, auch wenn keine Ophthalmoplegie vorliegt, auf ein zugrunde liegendes Guillain-Barré-Syndrom hinweisen [64]. Die Papillophlebitis wurde auch als erste Manifestation der Krankheit dokumentiert, die zu schwerwiegenden Komplikationen wie Blutungen des Sehnervs, Makulaödemen und Watteflecken führen kann [65].

Bis zu 50\% der Patienten haben eine Form der Hirnnervenbeteiligung [64], und Ophthalmoparese ist eine häufige okuläre Manifestation des Guillain-Barré-Syndroms. Lähmungen von Hirnnerven, in der Regel des dritten, sechsten und zwölften Hirnnervs, können zu Symptomen wie Esotropie, Augenschmerzen, Augenmuskelparese, Empfindlichkeit der Hornhaut, Lagophthalmus, Ektropium, eingeschränkter Augenlidbewegung und trockenem Auge führen [66]. Bei einigen Guillain-Barré-Patienten kann ein Papillenödem aufgrund von erhöhter Hirnflüssigkeit und Hirnödemen auftreten [67].

Aufgrund der Ähnlichkeit der klinischen Symptome, wie z.B. Anomalie der Augenlider und Pupillenfunktionsstörungen, kann das Miller-Fisher-Syndrom (MFS) fälschlicherweise für eine okuläre Myasthenia gravis gehalten werden [68], was eine angemessene Behandlung der okulären Manifestationen verzögert. Das MFS macht $5 \%$ bis $10 \%$ der Guillain-Barré-Fälle aus und ist durch eine Diplopie gekennzeichnet, die beim Guillain-Barré-Syndrom in der Regel fehlt und auf eine externe Ophthalmoplegie zurückzuführen ist [69]. MFS ist in Asien stärker verbreitet und macht allein in Japan bis zu einem Viertel der Guillain-Barré-Fälle aus [70]. Zu den weiteren okulären Manifestationen des Guillain-Barré-Syndroms gehören Blepharoptose, verschwommenes Sehen,
Photophobie, Lähmung des vertikalen Blicks, interne Ophthalmoplegie, abnorme Lidfunktion, Mydriasis, Anisokorie, Ptosis, Lidretraktion, Oberlidzuckungen, Lidnystagmus und Lagophthalmus. Es wurde auch über Farbenblindheit, verminderte Sehschärfe, Pupillenanomalien und supranukleäre Blicklähmung berichtet.

\section{Myasthenia gravis}

Myasthenia gravis ist eine chronische Erkrankung, die aufgrund der Zerstörung von Rezeptorzellen an der Nervenverbindung zu einer verminderten Signalübertragung in der Skelettmuskulatur führt. Infolgedessen sind Patienten mit Myasthenia gravis anfällig für die Entwicklung von Schilddrüsenerkrankungen, was das Risiko einer Schilddrüsen-Augenerkrankung weiter erhöht [71]. Die Prävalenz der Myasthenia gravis nimmt zu, wobei die weltweite Prävalenz derzeit auf 20 Personen pro 100000 Einwohner geschätzt wird [72]. In einer Studie wurde festgestellt, dass bei 82\% der untersuchten Patienten eine Augenschwäche das erste Symptom war [73].

Diplopie und asymmetrische extraokulare Beteiligung [74, 75] sind häufige erste Symptome der Myasthenia gravis, die auf eine Beteiligung der Augenmuskeln hinweisen, die als okuläre Myasthenia gravis (OMG) bezeichnet wird. Derzeit tritt OMG bei $10-40 \%$ der Patienten mit Myasthenia gravis auf [76]. Bei diesen Patienten kommt es zu Diplopie, Ptosis, pupillenaussparender Ophthalmoplegie, internukleärer Ophthalmoplegie und Schilddrüsen-Augenkrankheit. Ophthalmoparese kann bei allen extraokularen Muskeln auftreten und zu einer stark eingeschränkten Bewegung des Augapfels führen. In einer Kohortenstudie von Tang et al. wiesen knapp 70\% eine Diplopie und 25\% eine Ptosis bei der ersten Untersuchung auf. Bei allen 40 Patienten war der seitliche Rektusmuskel betroffen, was zu einer Einschränkung der Augenbewegung führte [73].

Bei 21\% der OMG-Patienten wurde ein trockenes Auge festgestellt [77], das auf Lagophthalmus und Orbicularis-Schwäche zurückzuführen ist, und auch Blepharitis tritt auf [74]. Obwohl OMG bei Myasthenia gravis häufiger vorkommt, bestätigen seltene Fallberichte Nystagmus und pseudo-intranukleäre Ophthalmoplegie [78]. Bei diesen Patienten ist die Adduktion eingeschränkt.

\section{Endokrine Autoimmunerkrankungen}

Bei endokrinen Autoimmunerkrankungen werden hormonproduzierende Drüsen vom Immunsystem angegriffen, was zu einer Unter- oder Überproduktion verschiedener Hormone führt, die zur Aufrechterhaltung der Homöostase benötigt werden.

\section{Diabetes mellitus Typ 1}

Bei Diabetes mellitus Typ 1 führt die Zerstörung der insulinproduzierenden Betazellen der Bauchspeicheldrüse dazu, dass der Blutzuckerspiegel nicht mehr kontrolliert werden kann, da Insulin die Aufnahme von Glukose in die Zellen signalisiert. Diabetes mellitus Typ 1 ist eine systemische Erkrankung, die zu schweren Komplikationen führen kann. Daher ist die Früherkennung der Schlüssel zur Verhinderung von Organschäden, einschließlich 
des Auges. Im Gegensatz zu Typ-2-Diabetes wird Typ-1-Diabetes oft schon in jungen Jahren diagnostiziert.

Im Jahr 2020 lag die weltweite Prävalenz von Typ-1-Diabetes bei 9,5\%. Allein in Großbritannien stieg die Prävalenz von Diabetes jedoch zwischen 1980 und 2014 um 40\%, wobei der Typ-1-Diabetes etwa 10\% dieser Fälle ausmacht [79].

Bei Patienten mit Typ-1-Diabetes wurden eine diabetische Retinopathie (DR), ein diabetisches Makulaödem (DME), Katarakte und ein Glaukom festgestellt, wobei die Diplopie im Frühstadium der Erkrankung häufiger auftritt [80]. Linsenveränderungen, die natürlicherweise mit dem Alterungsprozess auftreten, sind bei Diabetikern deutlicher erkennbar, ebenso wie ein geringerer Akkommodationsreflex [81]. Gefäßschäden sind in der PrädiabetesPhase möglicherweise nicht nachweisbar; daher kann sich eine Augenerkrankung entwickeln und fortschreiten, bevor sie überhaupt erkannt wird, wenn kein regelmäßiges ophthalmologisches Screening durchgeführt wird.

Ein frühzeitiges Screening auf DR ist für den Erhalt des Sehvermögens von entscheidender Bedeutung, da ein frühzeitiges Eingreifen nachweislich das Risiko der Entwicklung einer DR verringert [82] und eine Verschlechterung der Sehschärfe verhindern kann [83]. Da ein Drittel der Diabetespatienten über 40 Jahre eine DR entwickelt [84], könnte ein frühzeitiges und regelmäßiges ophthalmologisches Screening den Sehkraftverlust von Millionen von Menschen verhindern. Hammes et al. fanden heraus, dass 27,4\% einer Typ-1-Diabetes-mellitus-Kohorte eine Retinopathie entwickelten, davon waren $8 \%$ schwere Fälle. Nach einer Nachbeobachtungszeit von 40 Jahren wurde bei $84,1 \%$ der Patienten eine Retinopathie festgestellt, wobei 50,2\% schwere Fälle waren, was die potenziellen Auswirkungen von mangelndem Bewusstsein, fehlendem Screening und nicht rechtzeitiger Behandlung auf die Patienten verdeutlicht [85]. In ähnlicher Weise stellten Srinivasan et al. fest, dass sich bei $43 \%$ der untersuchten Kohorte die DR bei der Nachbeobachtung verschlimmerte [86].

Während die verminderte Sehschärfe stark mit dem Grad des Kapillarverlustes bei Patienten mit DR zusammenhängt, können diese Veränderungen asymptomatisch sein. Dies wird durch die Ergebnisse von Duet et al. [87] unterstützt: Von Patienten, die sich einer OCT-A- und OCT-Bildgebung (OCT = optische Kohärenztomografie) unterzogen, wiesen etwa $30 \%$ eine Verringerung der Gefäßdichte im tiefen Kapillarplexus auf, obwohl sie keine Symptome einer DR zeigten [87]. Dies zeigt, wie wichtig ein frühzeitiges Screening für alle Patienten ist.

Bei Patienten mit Diabetes mellitus Typ 1 ist die Wahrscheinlichkeit, ein Glaukom und einen grauen Star zu entwickeln, doppelt so hoch wie bei gesunden Personen [80], wobei die Inzidenzrate in der afroamerikanischen Bevölkerung höher ist [88]. Darüber hinaus haben Frauen ein höheres Risiko für Katarakte im Zusammenhang mit Typ-1-Diabetes [89, 90]. Auch bei 0,7-3,5\% der pädiatrischen Typ-1-Diabetiker [91] wird über das Auftreten von Katarakten berichtet, häufig innerhalb der ersten 6 Monate nach der Diagnose [92], die zum Verlust des Sehvermögens führen können. Aus diesem Grund sollten regelmäßige augenärztliche Un- tersuchungen durchgeführt werden, auch bei Personen, die keine offensichtlichen Kataraktsymptome aufweisen. Es fehlen jedoch eindeutige Informationen über das okuläre Screening bei Kindern mit Typ-1-Diabetes.

\section{Morbus Basedow}

Morbus Basedow (Graves' disease) tritt auf, wenn die Immunzellen die Homöostase der Schilddrüse angreifen und stören, was durch die Überproduktion von Schilddrüsenhormonen zu einer Hyperthyreose führt. Eine höhere Prävalenz von Morbus Basedow tritt in entwickelten Ländern auf, in denen Jod leicht verfügbar ist. Die Prävalenz von Morbus Basedow wird weltweit auf 2-3\% geschätzt [93]. Morbus Basedow wurde auch mit anderen Autoimmunerkrankungen wie Myasthenia gravis [94] in Verbindung gebracht, was das Risiko von okulären Manifestationen erhöht.

Bei bis zu 50\% der Patienten mit Morbus Basedow treten okuläre Manifestationen auf [95], einschließlich der Basedow-Ophthalmologie (GO; Graves' ophthalmology), die bei bis zu einem Drittel der Patienten auftritt und Symptome wie Diplopie und Fotophobie verursachen kann $[96,97]$. Obwohl GO-Symptome innerhalb von Monaten oder einigen Jahren nach der Erstdiagnose auftreten können [8], können sie oft schon vor der Diagnose des Morbus Basedow auftreten, was wiederum das Auge als Indikator für die zugrunde liegende Krankheit hervorhebt [98]. Weitere häufige Symptome sind die Einziehung der Oberlider, trockene Augen und Ptosis. Es wurde auch eine positive Korrelation zwischen erhöhtem Augeninnendruck (IOP) und Alter im Vergleich zur Krankheitsschwere berichtet [97]. Darüber hinaus kommt es bei $60 \%$ der Patienten mit Morbus Basedow ohne Ptosis zu einem erhöhten IOP beim Blick nach oben aufgrund eines vergrößerten unteren Rektus [8].

Ein Sehverlust bei GO ist selten, sollte aber nicht unterschätzt werden. Schwere Komplikationen wie dysthyreote Optikusneuropathie treten bei bis zu 5\% der GO-Patienten auf [99]. Zhang et al. dokumentierten den ersten bekannten Fall eines Basedow-Patienten mit schwerer Chemose und Bindehautprolaps. In der Regel ist ein chirurgischer Eingriff erforderlich, um den Druck zu mindern, der von den vergrößerten Augenmuskeln ausgeübt wird, die den Sehnerv zusammendrücken. In diesem Fall stellte die orbitale Strahlentherapie eine sichere und wirksame Behandlungsoption dar, da der Patient nicht für eine Operation infrage kam [100].

\section{Hashimoto-Thyreoiditis}

Die Hashimoto-Thyreoiditis tritt aufgrund eines Angriffs von Immunzellen auf die Schilddrüse auf und ist die Hauptursache für eine Hypothyreose in den Industrieländern [101]. Sie wird mit anderen Autoimmunkrankheiten wie rheumatoider Arthritis und Typ-1-Diabetes in Verbindung gebracht [102]. Es gibt Hinweise darauf, dass Augenprobleme in erheblichem Maße mit der Hashimoto-Thyreoiditis in Verbindung stehen; es sind jedoch noch viele groß angelegte Studien erforderlich, um diese ersten Ergebnisse verallgemeinern zu können. 
Obwohl die Schilddrüsen-assoziierte Ophthalmopathie (TAO) häufiger bei Morbus Basedow auftritt, wurde auch berichtet, dass sie bei 6\% der Patienten mit Hashimoto-Thyreoiditis auftritt. Alter, Krankheit und Raucherstatus gelten als TAO-Risikofaktoren [103]. Da die TAO häufiger bei Morbus Basedow auftritt, wurde ihre Assoziation mit der Hashimoto-Thyreoiditis in der wissenschaftlichen Forschung bisher vernachlässigt, und es gibt nur wenige Berichte in der Literatur. In mehreren Fallstudien wird über eine schwere TAO berichtet [104, 105], die mit einer Vergrößerung der Augenmuskeln zusammenhängt. Obwohl selten, kann TAO auch bei euthyreoten Patienten auftreten, was darauf hinweist, dass man sich beim Screening von Patienten nicht zu sehr zurückhalten sollte, da diese schweren Fälle zu einer Kompression des Sehnervs und damit zu einer Sehbehinderung führen können.

Bei Hashimoto-Patienten wurden auch Unterschiede oder Anomalien der Hornhaut festgestellt. Kirgiz et al. beobachteten in einer Kohorte mit Hashimoto-Thyreoiditis eine signifikant reduzierte Hornhauthysterese und einen erhöhten kornealen kompensierten IOD im Vergleich zur Kontrollgruppe. Die Verringerung der Hornhauthysterese erzeugt Druck auf den Sehnerv, was zu Komplikationen wie dem Glaukom führt [106].

In Fallstudien wird auch über das seltene Auftreten von schwerer, langfristiger Diplopie und Sehverlust bei Hashimoto-Patienten berichtet [107]. Bei einem Patienten, bei dem zuvor fälschlicherweise eine doppelte elevatorische Lähmung diagnostiziert worden war, trat im Laufe eines Jahres eine schwere Diplopie auf. Erst als ein positives Ergebnis eines Zwangstests, Bluttests und Ultraschalluntersuchungen auf eine Schilddrüsenunterfunktion hindeuteten, wurde Hashimoto-Thyreoiditis diagnostiziert. Die Diplopie wurde durch orales Prednisolon nicht behoben. Dieser Bericht zeigt, wie wichtig ein regelmäßiges Screening bei Patienten mit typischen TAO-Symptomen und atypischen Symptomen ist. Es stellt sich unweigerlich die Frage: Hätte ein rechtzeitigeres und gründlicheres Screening Fehldiagnosen vermieden und zu erfolgreicheren Behandlungsergebnissen geführt?

Eine Keratokonjunktivitis sicca trat bei bis zu 85\% der TAO-Patienten auf $[108,109]$, wobei zu den Risikofaktoren eine Proptose und ein erniedrigter Spiegel an freiem Thyroxin gehören, doch gibt es nur wenige Literaturberichte über diese Manifestation. Obwohl sie bei euthyreoten Patienten in milderer Form auftritt, gibt es keine Korrelation zwischen der Keratokonjunktivitis sicca und dem Schweregrad der Hashimoto-Thyreoiditis [109]. Insgesamt weisen Hashimoto-Patienten im Vergleich zu gesunden Kontrollgruppen eine deutlich höhere Prävalenz des trockenen Auges auf, einschließlich einer wesentlich geringeren durchschnittlichen Tränenproduktion und Tränenstabilität. Zu den weiteren berichteten Manifestationen gehören Lidretraktion, Weichteilödeme, Proptosis und extraokuläre Beteiligung [110]. In dieser Studie wurden eine Keratokonjunktivitis sicca, eine deutlich geringere Tränenproduktion und Tränenstabilität, eine Funktionsstörung der Meibom-Drüsen, eine Proptose und eine verringerte Becherzelldichte festgestellt. Der durchschnittliche Flächenverlust der Meibom-Drüsen lag bei $25 \%$, bei einem Patienten sogar bei $50 \%$.

\section{Dermatologische Autoimmunerkrankungen}

Dermatologische Autoimmunerkrankungen führen in der Regel zu gereizten oder blasigen Hautstellen am ganzen Körper, die für den Patienten unangenehm sind. Erkrankungen wie die systemische Sklerose betreffen vor allem die Haut. Häufig sind jedoch auch andere Organe von der Krankheit betroffen, darunter auch das Auge.

\section{Psoriasis}

Bei der Psoriasis kommt es zu einem beschleunigten Wachstum der Hautzellen, was zu roten, schuppigen Läsionen am ganzen Körper führt. Derzeit leben bis zu 3\% der Weltbevölkerung mit Psoriasis [111]. In Norwegen wurden Prävalenzschätzungen von bis zu 11,4\% gemeldet, und die Prävalenz nimmt zu [112].

Die Prävalenz der okulären Manifestationen ist umstritten, aber bis zu 70\% der Patienten weisen eine okuläre Assoziation auf [113]. Eine positive Korrelation zwischen dem Schweregrad der Exazerbationen der Haut und den okulären Manifestationen wurde ebenfalls festgestellt [113]. Chowdhury et al. fanden heraus, dass fast die Hälfte der gescreenten Patienten mit okulären Manifestationen unauffällige Symptome haben oder asymptomatisch sind [114], was darauf hindeutet, dass Patienten unabhängig von Risikofaktoren oder bei Asymptomatik regelmäßig augenärztlich untersucht werden sollten, um eine frühzeitige Behandlung zu ermöglichen. Am Auge kann die Psoriasis das Augenlid, die Bindehaut und die Hornhaut betreffen [111].

Eine chronische Konjunktivitis betrifft etwa 64,5\% der Patienten $[111,115,116]$. In der Bindehaut können Bindehautläsionen, Xerose, Symblepharon und Trichiasis auftreten. Chronische Blepharitis ist ebenfalls häufig und führt zu Ektropium, Trichiasis, Madarose, Verlust von Gewebe am Augenlid und Sehstörungen [117], am häufigsten bei jüngeren Patienten [118]. Erbagci et al. fanden bei $67,74 \%$ der Plaque-Psoriasis-Patienten mindestens eine Anomalie des vorderen Abschnitts. Die chronische Blepharokonjunktivitis war bei $64,5 \%$ der Patienten die wichtigste Augenerkrankung. Es wurde auch über Hornhauttrübungen, Katarakte und Pigmentstörungen der Hornhaut berichtet [119].

Eine trockenes Auge tritt Berichten zufolge bei 18,75\% der Patienten mit Psoriasis auf [111], wobei dieser Wert je nach Studie variiert. Im Allgemeinen ist eine Beteiligung der Hornhaut selten; in einem Bericht wurde jedoch über ein peripheres Schmelzsyndrom der Hornhaut (PCMS) bei einem Patienten berichtet. In diesem Fall trat ein schweres Hornhautgeschwür auf, bei dem $90 \%$ der Hornhautdicke betroffen waren. Trotz des möglichen Sehkraftverlusts lassen sich die Augenprobleme mit einer aggressiven Behandlung beheben [120].

Bei bis zu 20\% der Patienten wurde eine Uveitis festgestellt [121], wobei es sich in der Regel um eine pustulöse Form handelt. Es wurden Zusammenhänge zwischen Psoriasis und DR festgestellt [122]. Darüber hinaus berichteten Ajitsaria et al. über den ersten bekannten Fall einer rezidivierenden orbitalen Myositis in Verbindung mit juveniler Psoriasis, die zu einer dauerhaften Sehbehinderung führte [123].
10

Kompass Ophthalmol 2022;8:2-16 DOI: $10.1159 / 000521961$ 


\section{Systemische Sklerose}

Die systemische Sklerose oder Sklerodermie betrifft weltweit bis zu 2,5 Millionen Menschen [124] und tritt entweder als lokalisierte Form auf, bei der nur die Haut betroffen ist, oder als systemische Form, bei der mehrere Organe betroffen sind. Bei systemischer Sklerose führt die beschleunigte Kollagenproduktion zu einer harten, dicken Haut sowie zu Schwellungen und Gelenkschmerzen. Darüber hinaus wird Sklerodermie auch mit anderen Autoimmunkrankheiten wie rheumatoider Arthritis und dem Sjögren-Syndrom in Verbindung gebracht [125]. Allein in Großbritannien leben fast 20000 Menschen mit systemischer Sklerose, und bis zum Jahr 2038 wird ein Anstieg der Prävalenz um 26\% prognostiziert [126].

Bei systemischer Sklerose treten zahlreiche Manifestationen des Auges mit unterschiedlichem Schweregrad auf, darunter retinale Anomalien, Katarakte, Blepharitis, Teleangiektasien, sklerale Grübchen und Keratokonus [127, 128]. In einer Studie war der häufigste Befund das trockene Auge (64,71\%), gefolgt von Hautveränderungen der Augenlider (56,86\%) und retinalen Anomalien $(50,98 \%)$. Die Patienten haben oft eine reduzierte Gefäßversorgung, was zu einer möglichen Aderhautatrophie führt [129]. In einer kleinen Studie von Grennan et al. traten bei diesen Patienten zwar nicht häufig trockene Augen und retinale Anomalien auf, aber 50\% der Patienten wiesen Anomalien der Aderhaut auf, bei denen es sich um Bereiche ohne Perfusion handelte [130]. Ein Patient mit Keratokonus wies eine verringerte Hornhautdicke, eine schwere Keratokonjunktivitis sicca und eine verringerte Tränenstabilität zusätzlich zur Hornhautverkrümmung auf [131]. IrisTransillumination tritt auf, wenn die Sklerodermie die Pigmente der Iris beeinträchtigt [132]. Bei Sklerodermie-Patienten wurde festgestellt, dass die subfovealen Aderhautschichten dünner sind. Über die Hälfte der Patienten mit systemischer Sklerose leidet an trockenen Augen [133]. Horan fand heraus, dass, obwohl nur ein Drittel ihrer Kohorte unter trockenen Augen litt, fast die Hälfte über eine verringerte Tränensekretion berichtete, was auf eine mögliche Entwicklung zu einer Keratokonjunktivitis sicca hindeutet [134]. Eine Fibrose der Tränendrüse, eine chronische Blepharitis und eine Funktionsstörung der Meibom-Drüsen können $\mathrm{zu}$ einem trockenen Auge führen. In früheren Berichten wurde festgestellt, dass bei bis zu 65\% der untersuchten SklerodermiePatienten eine Lidsteifigkeit auftritt [125], die auf eine Fibrose der Augenlider zurückzuführen ist. Diese Steifheit der Augenlider kann zu einem Lagophthalmus führen, der die Patienten anfällig für Schäden oder Infektionen macht [132].

Bei der begrenzten Form der Krankheit treten auch okuläre Manifestationen auf. ECS (Sklerodermie en coup de sabre) weist zahlreiche Augensymptome auf, darunter Ptosis, Uveitis, trockenes Auge, Episkleritis, orbitale Myositis [135] und eine reduzierte Größe des Augapfels und der Augenmuskeln [136]. Die meisten bekannten Berichte über Augensymptome bei ECS traten bei $26 \%$ der Patienten auf. Es wurden Keratopathie, Netzhautablösung, eingeschränkte Augenbeweglichkeit, Diplopie, Katarakte und Hornhautastigmatismus beobachtet [136]. Obwohl selten, wurde eine Adie-Pupille als initialer Krankheitsindikator für ECS be- richtet [135]. Die lineare Sklerodermie ist bei Kindern häufiger zu beobachten [137], wobei okuläre Manifestationen beim ESC-Subtyp bei pädiatrischen Patienten häufiger vorkommen [138]. Die lineare ECS wurde auch mit retinalen Teleangiektasien und exsudativen Netzhautablösungen [137] sowie Uveitis [138] in Verbindung gebracht.

\section{Dermatomyositis}

Dermatomyositis ist die häufigste Form der idiopathischen entzündlichen Myopathie, die darauf zurückzuführen ist, dass das Immunsystem die Muskelgefäße und das Bindegewebe des Körpers angreift. $\mathrm{Zu}$ den charakteristischen Symptomen gehören rote, schuppige Hautflecken und Gottron-Papeln. Außerdem können ein heliotroper Ausschlag am oberen Augenlid und Ödeme um die Augen auftreten. Von Dermatomyositis sind schätzungsweise 9,6 pro 1 Million Menschen betroffen, wobei das Risiko für Frauen [139] und Afroamerikaner [140] am größten ist. Am häufigsten sind Erwachsene zwischen 40 und 60 Jahren und Kinder zwischen 5 und 15 Jahren betroffen, wobei Kinder im Allgemeinen eine akutere Erkrankung erleiden [141].

Die okulären Manifestationen der Dermatomyositis sind zahlreich und umfassen heliotropen Ausschlag, Ödeme um die Augen, Ptosis, Diplopie, verschiedene Schielerkrankungen, Bindehautödeme, Nystagmus, Iritis, Watteflecken, Optikusatrophie, Pseudopolyposis der Bindehaut, Linsenanomalien, Episkleritis und Uveitis mit Glaukom [142]. Es wurde auch über internukleäre Ophthalmoplegie berichtet [143]. Außerdem wurde über irreversible Sehstörungen infolge okulärer Manifestationen der Dermatomyositis berichtet [144, 145]. Die systemische Verabreichung von Methylprednisolon und Cyclophosphamid konnte den durch eine Schädigung der Makula und des Sehnervenkopfes verursachten Sehverlust bei einer Patientin nicht beheben. Auch hier stellt sich die Frage nach einem früheren Eingreifen und der Möglichkeit, eine dauerhafte Sehbehinderung zu vermeiden [144].

Seltene Fälle von akutem retinalem Zentralarterienverschluss (ZAV) im Zusammenhang mit Dermatomyositis sind in der Literatur beschrieben worden [146]; sie äußern sich durch Symptome wie Papillenödeme und peripapilläre Blutungen, die umfangreiche Netzhautschäden verursachen. In diesem Fall wurde eine Ischämie des Sehnervs für die ZAV-Präsentation verantwortlich gemacht. Ein Sehverlust bei ZAV ist zwar selten, kommt aber vor. Darüber hinaus treten bei einem ZAV, der länger als 240 Minuten andauert, erhebliche irreversible Schäden auf [147], was die Bedeutung des Bewusstseins für die Krankheit und einer rechtzeitigen Behandlung unterstreicht.

Die orbitale Myositis ist eine weitere seltene, aber schwere Manifestation der Dermatomyositis. Der erste bekannte Bericht über dieses Vorkommnis stammt aus dem Jahr 2005, als ein männlicher Patient Muskelschmerzen gefolgt von Augenlidödemen und Augenschmerzen hatte, zusammen mit einer nicht diagnostizierten Dermatomyositis. Die Magnetresonanztomografie zeigte eine beidseitige Vergrößerung des lateralen und inferioren Rektusmuskels, die erfolgreich mit einer Immunglobulintherapie behandelt wurde [148]. 


\section{Verschiedene Autoimmunerkrankungen}

ANCA-Vaskulitis. Die mit antineutrophilen zytoplasmatischen Antikörpern (ANCA) assoziierte Vaskulitis kann verschiedene Organe des Körpers betreffen und ist in erster Linie durch eine Entzündung der Blutgefäße gekennzeichnet, die zu deren Zerstörung führt. ANCA zielen auf das Zytoplasma von Neutrophilen $a b$, und ihre Anhaftung führt zur Auslösung eines neutrophilen Angriffs auf die Blutgefäße. Dies führt zu Schwellungen und Entzündungen von Blutgefäßen in verschiedenen Körperregionen. In Europa erkranken jährlich zwischen $20-25$ pro 1 Million Menschen [149].

Eine solche Erkrankung, die unter ANCA-assoziierte Vaskulitis fällt, ist die Granulomatose mit Polyangiitis (GPA). Diese Erkrankung ist gekennzeichnet durch eine Vaskulitis der kleinen und mittleren Blutgefäße mehrerer Organsysteme, einschließlich der Nase, der Nieren und des Atmungssystems. Nach Angaben der National Organization for Rare Disorders treten bei mehr als der Hälfte der GPA-Patienten okuläre Symptome auf (z.B. Bindehautentzündung, Hornhautgeschwüre, Schmerzen, Entzündung der Sklera und Sehkraftverlust). In einigen Fällen können sie als erstes Symptom einer GPA auftreten [150].

In einer kürzlich veröffentlichten Fallstudie sprach eine 47-jährige Frau nicht auf entzündungshemmende und antimikrobielle Therapien zur Behandlung langfristiger bilateraler Augensymptome (Skleraentzündung, Sekundärglaukom und Hornhautgeschwür) an. Ein Hornhautgeschwür stellte sich in diesem Fall als erstes Symptom von GPA dar, das später durch Gewebeproben diagnostiziert wurde. Außerdem öffnete sich der Abszess der Bindehaut und legte die Sklera frei. Der Patient zeigte ein erfolgreiches Ergebnis bei der Behandlung mit Zytostatika und Prednisolon. Die Hornhaut- und Bindehautdefekte blieben jedoch bestehen, was das Potenzial für langfristige, anhaltende okuläre Manifestationen im Zusammenhang mit dieser Autoimmunerkrankung deutlich hervorhebt [151].

\section{Erkrankung bedingt durch Immunglobulin G4}

Die Immunglobulin-G4 (IgG4)-assoziierte Erkrankung ist eine seltene und chronische fibro-inflammatorische Erkrankung, die durch erhöhte IgE- und IgG4-Spiegel gekennzeichnet ist. Es handelt sich um eine systemische Krankheit, die mehrere Körperorgane betreffen kann, darunter die Augen, die Lunge und die Nieren. Wenn die Krankheit das Auge betrifft, spricht man von einer orbitalen IgG4-bedingten Krankheit, bei der es zu lymphoplasmatischen Infiltrationen der adnexalen Gewebe des Auges (z.B. der Tränendrüsen und Augenlider) kommt. IgE-Level steigen durch eine Überproduktion von Zytokinen, z.B. Interleukin (IL)4 und IL-13, während IgG4 durch eine erhöhte Produktion von IL-10 ansteigt. Insgesamt führt dies zu einer fibrogenen ZytokinTGF- $\beta$-Produktion, die zu Fibrose führt [152].

Goto et al. führten eine retrospektive multizentrische Studie durch, um die klinischen Symptome von Personen mit orbitaler IgG-bedingter Erkrankung zu bewerten. Bei der Analyse von 378 Patienten in 9 japanischen Zentren ergaben bildgebende Untersuchungen das Auftreten von Augenläsionen im Zusammenhang mit der orbitalen IgG4-bedingten Erkrankung, die verschiedene Augengewebe betreffen, darunter die Augenlider (12\%) und am häufigsten die Tränendrüse (86\%). Interessanterweise traten bei knapp der Hälfte der Patienten Läsionen des extraokularen Gewebes auf, was deutlich macht, dass es sich zwar um eine seltene Krankheit handelt, verheerende okuläre Manifestationen jedoch häufig vorkommen. Das am häufigsten berichtete Augensymptom war das trockene Auge, das bei $22 \%$ der Patienten auftrat. Weitere Symptome wie Diplopie und Sehstörungen wurden ebenfalls berichtet [153].

\section{Cogan-Syndrom}

Das Cogan-Syndrom ist eine seltene Krankheit mit nur etwa 250 bekannten Fällen [154], bei der es zu einer Zerstörung von Gewebe im Ohr und in den Augen infolge eines Angriffs des Immunsystems kommt. Der Mechanismus dieser Krankheit ist weitgehend unbekannt; es ist jedoch bekannt, dass die Patienten infolge dieser Gewebezerstörung unter Augensymptomen wie Reizungen und Sehstörungen leiden.

Migliori et al. berichteten über den Fall einer 31-jährigen Frau, die neben einer beidseitigen Bindehautentzündung auch Ohrensymptome wie Hörverlust und Tinnitus aufwies. Nach 10 Tagen sprach die Patientin nicht mehr auf die anfängliche Behandlung an und ihr Zustand verschlechterte sich, sodass die Behandlungsstrategie auf ein systemisches Kortikosteroid umgestellt wurde. 10 Tage nach Beginn der systemischen Prednisolon-Behandlung traten bei der Patientin schwerere Augensymptome auf, darunter Tränenfluss und Photophobie, sowie weitere Symptome, die die Ohren betrafen, wie z.B. sensorineuraler Hörverlust. Darüber hinaus wurde bei einer weiteren augenärztlichen Untersuchung eine interstitielle Keratitis festgestellt, was die Diagnose des Cogan-Syndroms ermöglichte. Die okulären Symptome verschwanden 4 Monate nach Beginn der Behandlung mit Immunsuppressiva [155].

\section{Epidemiologische Faktoren, die die Prävalenz von Augen- komplikationen bei Autoimmunerkrankungen beeinflussen}

\section{Geschlechtsspezifische Unterschiede bei der Prävalenz ver-} schiedener Autoimmunkrankheiten

Bei vielen Autoimmunkrankheiten sind geschlechtsspezifische Unterschiede sowohl bei der Prävalenz als auch bei der Schwere der Erkrankung gut dokumentiert. Bei den mehr als 80 existierenden Autoimmunerkrankungen machen Frauen schätzungsweise bis zu 85\% der Patienten aus [156] und haben ein höheres Risiko für eine Polyautoimmunität [157]. Eine solche Tendenz könnte durch die Genetik beeinflusst sein. Das X-Chromosom enthält 10\% der körpereigenen miRNA [158]. Bei Frauen werden Gene wie KDM6A, die auf dem X-Chromosom liegen, stärker exprimiert, was zu Immunreaktionen führt, wenn Anomalien wie eine unvollständige Aktivierung auftreten [159]. Eine höhere Expression des Transkriptionsfaktors VGLLL3 bei Frauen wurde auch stark mit dem Sjögren-Syndrom und der systemischen Sklerose in Verbindung gebracht [160] - zwei Krankheiten, bei denen das weibliche Geschlecht stärker betroffen ist. 
Die Genetik beeinflusst auch die Schwere der Erkrankung. Während Frauen im Allgemeinen eine chronische, fibrotische und Th2-dominierte Immunantwort haben, könnte die charakteristische akute Entzündung bei Autoimmunkrankheiten mit männlichem Bias auf ihre vorherrschende Th1-Immunantwort zurückzuführen sein [161]. Dies könnte eine Erklärung dafür sein, warum Frauen häufiger erkranken, während bei Männern die Krankheit oft schwerer verläuft. Dafür spricht, dass akute, antikörpervermittelte Krankheiten wie Morbus Basedow und Myasthenia gravis häufiger bei Frauen auftreten, während akute Entzündungskrankheiten häufiger bei Männern zu finden sind.

Auch das verschiedene hormonelle Milieu von Männern und Frauen kann dazu beitragen. Testosteron und Progesteron sollen, je nach Level, immunsuppressive Wirkungen haben, während Östrogen und Prolaktin eine Polarisierung der Immunantwort in Richtung Th2-Antwort bewirken und höhere Antikörperspiegel erzeugen [162]. Darüber hinaus können die endokrinen Umstellungen, die Frauen in mindestens zwei Lebensabschnitten durchlaufen, eine erhöhte Anfälligkeit für die Ausprägung einer Veranlagung und den Schweregrad von Krankheiten verursachen.

\section{Rheumatologie}

Frauen sind bei rheumatologischen Autoimmunerkrankungen wie rheumatoider Arthritis und Sjögren-Syndrom stärker betroffen. So machen Frauen mittleren Alters in den meisten untersuchten Patientenkohorten über 70\% der Fälle von rheumatoider Arthritis aus [163] und sie haben ein höheres Risiko für ein trockenes Auge [139]. Andererseits ist die Prävalenz von Morbus Behçet bei Männern höher, mit einem größeren Risiko für okuläre Manifestationen [164].

\section{Magen-Darm}

Geschlechtsspezifische Unterschiede bei CED sind in der Literatur umstritten. Die relative Prädisposition zwischen den Geschlechtern blieb bei Colitis ulcerosa bis zum Alter von 45 Jahren ungefähr gleich, wonach Männer eine höhere Prädisposition für die Krankheit entwickelten [165]. Widersprüchlichen Berichten zufolge haben Frauen ein 20-30\% höheres Risiko, an Colitis ulcerosa zu erkranken als Männer [32]. Augensymptome treten bei Frauen mit Morbus Crohn häufiger auf [166]. Was die Zöliakie betrifft, so haben neuere Studien ein erhöhtes Risiko für eine nicht diagnostizierte Zöliakie bei Mädchen und Frauen festgestellt [167], Nijhawan et al. weisen jedoch auf eine höhere Inzidenz bei Männern hin [168].

\section{Neurologisch}

Es gibt Hinweise auf geschlechtsspezifische Unterschiede bei der Ausprägung der Anfälligkeit für neurologische Autoimmunerkrankungen. Bei der entzündlich geprägten multiplen Sklerose besteht eine höhere weibliche Prädisposition, jedoch sind Krankheitsverlauf und Schweregrad bei Männern schlechter [159]. Forschungsergebnisse deuten darauf hin, dass die Schmerzen bei weiblichen MS-Patienten auf ein reaktiveres Immunsystem zu- rückzuführen sind, während bei Männern die Manifestation der Schmerzen aufgrund der schnellen Neurodegeneration größer ist [169].

Myasthenia gravis kann in jedem Alter auftreten, jedoch sind jüngere Frauen und ältere Männer einem höheren Risiko ausgesetzt [170]. Außerdem ist das Risiko, an OMG zu erkranken, bei Männern doppelt so hoch [171].

\section{Endokrin}

Endokrine Autoimmunerkrankungen wie die Hashimoto-Thyreoiditis sind mit Ausnahme des Typ-1-Diabetes häufiger bei Frauen anzutreffen [102]. Da die Insulinsensitivität bei Frauen höher ist, könnte dies ein Faktor sein, der die höhere Prävalenz der Krankheit bei Männern erklärt [172]. Trotz des männlichen Bias bei Typ-1-Diabetes haben Frauen ein um 30\% höheres Risiko für eine Retinopathie als Manifestation der Krankheit und für erhöhte Komplikationen während der Schwangerschaft [173, 174].

\section{Dermatologie}

Frauen erkranken häufiger an einer begrenzten Sklerodermie als Männer, vor allem in jüngerem Alter. Männer neigen eher dazu, die systemische Sklerose in einem höheren Alter zu entwickeln [175]. Obwohl die Berichte über geschlechtsspezifische Unterschiede widersprüchlich sind, deuten neuere Studien darauf hin, dass die Psoriasis bei Männern signifikant schwerer verläuft und häufiger zu Augenkomplikationen führt [176]. Obwohl Frauen ein höheres Risiko haben, an Dermatomyositis zu erkranken [139], fanden Tseng et al. heraus, dass Männer mit Dermatomyositis im Vergleich zu Frauen eher am Sjögren-Syndrom erkranken [177]. Die Dermatomyositis zeigt eine etwas höhere weibliche Prädisposition, die jedoch mit dem Alter natürlich abnimmt [178].

\section{Auswirkung eines hormonellen Ungleichgewichts auf die} Prävalenz verschiedener Autoimmunkrankheiten

\section{Pubertät}

Vor der Pubertät sind die Geschlechtsunterschiede zwischen den Kindern weniger bedeutsam sowohl für die Inzidenz als auch für den Schweregrad, da das hormonelle Milieu in dieser frühen Lebensphase ähnlich ist. Da es jedoch nur wenige Informationen über Autoimmunkrankheiten im Kindesalter gibt, ist es schwierig, Muster zu erkennen.

Beim Behçet-Syndrom in der Vorpubertät werden keine geschlechtsspezifischen Unterschiede beobachtet [178]. Ähnliche Muster sind bei jungen Frauen mit MS zu beobachten, wobei sich das Risiko für Frauen im Vergleich zu Männern im Alter von 13 Jahren verdoppelt [179]; allerdings treten Krankheitsprogression und Komplikationen bei Männern schneller auf. Darüber hinaus nehmen einige Krankheiten während der Pubertät an Schwere zu, wie z.B. Typ-1-Diabetes aufgrund einer verminderten Insulinresistenz [180]. Daher sollten sich die Ärzte der möglichen schweren okulären Manifestationen bewusst sein, die sich aus einer Verschlechterung des Krankheitszustands ergeben können. 


\section{Schwangerschaft}

Obwohl es unterschiedliche Berichte gibt, wurde allgemein festgestellt, dass Schwangerschaft und Langzeitstillen Krankheiten wie Psoriasis [181] und rheumatoide Arthritis [182] aufgrund erhöhter entzündungshemmender Östradiolspiegel lindern können. Andererseits erhöht eine Schwangerschaft das Risiko, an multipler Sklerose zu erkranken, weiter [183].

Obwohl sich einige Autoimmunkrankheiten während der Schwangerschaft verschlimmern, ist der Körper insgesamt bestrebt, das Immunsystem der Mutter zu unterdrücken, um den Fötus zu schützen, indem er zu einer Th2-Immunantwort übergeht, was die beobachteten Remissionsphasen bei einigen Autoimmunkrankheiten und ihren okulären Manifestationen während der Schwangerschaft erklärt [184].

\section{Wechseljahre}

Einige Krankheiten wie rheumatoide Arthritis und Schuppenflechte können sich nach den Wechseljahren verschlimmern, was wahrscheinlich auf die Veränderungen im körpereigenen Immunsystem und den höheren Gehalt an entzündungsfördernden Zytokinen zurückzuführen ist. Außerdem wurde bei Männern mit rheumatoider Arthritis ein erhöhter Östrogenspiegel festgestellt [185]. Das durchschnittliche Höchstalter für das Auftreten der rheumatoiden Arthritis fällt mit dem Alter der Wechseljahre zusammen, obwohl ein früherer Beginn der Wechseljahre mit einer weniger schweren rheumatoiden Arthritis in Verbindung gebracht wurde [186]. Einige Krankheitsverläufe können durch die Wechseljahre nicht beeinflusst werden. Jüngste Daten deuten beispielsweise darauf hin, dass sich der Schweregrad von Morbus Crohn und Colitis ulcerosa in den Wechseljahren nicht verändert, sondern sogar verbessert [187].

\section{Geografische und ethnische Unterschiede}

Wie in den vorangegangenen Abschnitten erwähnt, ist die Anfälligkeit für bestimmte Autoimmunkrankheiten auf eine genetische Veranlagung und Umweltfaktoren zurückzuführen. Epidemiologische Daten deuten auf offensichtliche ethnische und geografische Unterschiede bei mehreren der in dieser Übersicht behandelten Autoimmunkrankheiten hin. Insgesamt weist die afroamerikanische Bevölkerung eine höhere Prävalenz von Autoimmunerkrankungen auf. Es gibt jedoch keine ausreichenden Informationen, um festzustellen, ob dies auf genetische Unterschiede, Umweltfaktoren oder beides zurückzuführen ist. Die Identifizierung derjenigen, die am meisten gefährdet sind, bestimmte Autoimmunkrankheiten $\mathrm{zu}$ entwickeln und somit auch die damit verbundenen okulären Manifestationen, kann dazu beitragen, das Bewusstsein der Kliniker und Forscher zu schärfen.

\section{Rheumatologie}

Rheumatoide Arthritis kommt in den westlichen Ländern häufiger vor, was wahrscheinlich auf Umweltfaktoren zurückzuführen ist, z.B. auf das häufigere Rauchen. Es gibt nur wenige Informationen über die Prävalenz der rheumatoiden Arthritis in Entwick- lungsländern. Was die ethnischen Gruppen anbelangt, so haben afroamerikanische Patienten ein etwas höheres Risiko, an rheumatoider Arthritis zu erkranken und unter der Behandlung mit krankheitsverändernden Antirheumatika (DMARD) eine geringere Remissionsrate zu erzielen [188]. Was die pädiatrische Bevölkerung mit rheumatoider Arthritis betrifft, so ist die Prävalenz von Kindern, die rheumatologische Kliniken aufsuchen, in südasiatischen Ländern höher als in westlichen Ländern, mit Ausnahme von Singapur [189]. Die meisten Berichte befassen sich mit Fällen in westlichen Ländern; dies ist jedoch die erste Studie über Südostasien.

Interessanterweise fanden Muro et al. bei 314 japanischen Patienten mit systemischer Sklerose und Sjögren-Syndrom [190] niedrigere Konzentrationen von Anti-NT5C1A, einem Autoantikörper, der mit einem schweren Krankheitsverlauf in Verbindung gebracht werden kann, was möglicherweise darauf hindeutet, dass japanische Patienten im Allgemeinen einen weniger schweren Krankheitsverlauf dieser Krankheiten haben könnten. Die Erkenntnis, dass systemische Sklerose in Europa, Kanada und Nordamerika häufiger vorkommt, bestätigt diese Ergebnisse. Es sind jedoch umfangreichere Studien erforderlich, um festzustellen, ob dieses Ergebnis auf die Gesamtbevölkerung verallgemeinert werden kann. Darüber hinaus haben groß angelegte Studien ergeben, dass das Risiko, am SjögrenSyndrom zu erkranken, bei afroamerikanischen Patienten geringer ist [191].

Die höchste Prävalenz von Morbus Behçet findet sich im Mittelmeerraum [25]. In der Türkei liegt die geschätzte Prävalenz bei 421 pro 100000 Einwohner [26]. Bei Afrikanern südlich der Sahara wird ein schwererer Krankheitsverlauf festgestellt [192].

\section{Magen-Darm}

Die Prävalenz von Morbus Crohn und Colitis ulcerosa bei verschiedenen ethnischen Gruppen variiert zwischen den Ländern. Es gibt nur wenige Daten über die Prävalenz von Krankheiten in Ländern der Dritten Welt. Die weltweit höchste Prävalenz von Morbus Crohn und Colitis ulcerosa wird in westlichen Ländern beobachtet, nämlich in Kanada für Morbus Crohn und in den USA, Dänemark und Island für Colitis ulcerosa [193], weshalb die meisten Studien aus diesen Ländern stammen. In einer kürzlich in Großbritannien durchgeführten Studie wies eine indische Kohorte das höchste Risiko auf, an Colitis ulcerosa zu erkranken [194], während andere Studien zeigen, dass Menschen aus Südasien ein höheres Risiko haben, sowohl an Morbus Crohn als auch an Colitis ulcerosa zu erkranken [195]. Colitis ulcerosa nimmt in Asien zu, was darauf hindeutet, dass CED eine globale Krankheit und keine Krankheit der westlichen Länder ist [196].

In den USA sind weiße und indianische Bevölkerungsgruppen stärker betroffen als afroamerikanische, hispanische und ostasiatische Bevölkerungsgruppen [197, 198]. Eine Studie mit 228 Patienten in San Francisco ergab, dass asiatische und hispanische Kohorten im Vergleich zu schwarzen und weißen Kohorten häufig in einem späteren Alter diagnostiziert wurden, was sich auf den Krankheitsverlauf und die damit verbundenen okulären 
Tab. 3. Augennebenwirkungen verschiedener Medikamente zur Behandlung von Autoimmunerkrankungen

\begin{tabular}{|c|c|c|}
\hline Medikament & Assoziierte okuläre Wirkung & Referenz \\
\hline Methotrexat & $\begin{array}{l}\text { Bindehautentzündung, trockenes Auge, Blepharitis, Watteflecken, Photophobie, Epiphora, Optikusneuropathie, } \\
\text { Augenbrennen, Reizung und verschwommenes Sehen }\end{array}$ & {$[221-223]$} \\
\hline Azathioprin & retinale Vaskulitis, Cytomegalovirus (CMV)-Retinitis und Uveitis & {$[224]$} \\
\hline Hydroxychloroquin & Netzhauttoxizität, Bull's-Eye-Makulopathie und Wirbelkeratopathie & {$[225,226]$} \\
\hline Cyclooxygenase-Hemmer & Bindehautentzündung, verschwommenes Sehen, verzweigte retinale Venenverschlüsse und Thrombosen & {$[227,228]$} \\
\hline Glukokortikoide & $\begin{array}{l}\text { subkapsulärer Katarakt, sekundäres Offenwinkelglaukom, Katarakt, Schädigung des Sehnervs, Mydriasis, } \\
\text { Chororetinopathia centralis serosa }\end{array}$ & {$[229,230]$} \\
\hline Anti-TNFa-Medikamente & $\begin{array}{l}\text { Uveitis, Vitritis, orbitales Granulom, orbitale Myositis, Herpes-Zoster-Keratitis, Skleritis, Sehnervenentzündung und } \\
\text { Chiasmopathie }\end{array}$ & {$[231]$} \\
\hline Aminosalicylate & trockenes Auge, verschwommenes Sehen, Steven-Johnson-Syndrom und Optikusneuropathie & {$[232,233]$} \\
\hline $\begin{array}{l}\text { Psoralen und Ultraviolett A } \\
\text { (PUVA) }\end{array}$ & Hyperämie der Bindehaut, verminderte Linsentransparenz, trockenes Auge, Kataraktbildung und Linsentrübungen & {$[234-236]$} \\
\hline
\end{tabular}

Manifestationen auswirken könnte. Außerdem war Morbus Crohn in der schwarzen ethnischen Gruppe im Vergleich zur asiatischen Gruppe am häufigsten [199]. Afroamerikaner haben ein höheres Risiko, okuläre Manifestationen wie Uveitis zu entwickeln [193].

Wie bei vielen Autoimmunkrankheiten ist die Prävalenz der Zöliakie in den einzelnen Ländern unterschiedlich. Die Zöliakie hat eine hohe Prävalenz in Nordamerika, Nordafrika, dem Nahen Osten und Indien, aber eine niedrige Prävalenz in Afrika südlich der Sahara und Ostasien [200, 201]. So wurde beispielsweise in nördlicheren Regionen der Vereinigten Staaten eine höhere Prävalenz von Zöliakiepatienten festgestellt als in südlichen Regionen [202]. Okuläre Manifestationen treten eher bei erwachsenen Zöliakiepatienten als bei Kindern auf [203].

\section{Neurologisch}

Die Prävalenz der multiplen Sklerose ist in Nordamerika, Westeuropa und Australasien höher als in Afrika und Ozeanien [204]. In Großbritannien wurde in einer groß angelegten Studie festgestellt, dass die BAME-Bevölkerung im Vergleich zu den Weißen ein höheres Risiko hat, an MS zu erkranken, und dass auch der Raucherstatus eine Rolle spielt, obwohl das Durchschnittsalter bei der Diagnose ähnlich ist, mit Ausnahme der Südasiaten [205]. Die geografische Prävalenz des Guillain-Barré-Syndroms wird durch saisonale Veränderungen beeinflusst. Insgesamt tritt das Guillain-Barré-Syndrom im Winter häufiger auf, insbesondere in den westlichen Ländern, im Fernen und Nahen Osten. Auf dem indischen Subkontinent und in Lateinamerika ist die Inzidenz im Winter dagegen geringer [206]. Die Demografie der Guillain-Barré-Subtypen ist weltweit sehr unterschiedlich.

Derzeit leiden in den USA mindestens 64000 Menschen an Myasthenia gravis [76]. Untersuchungen in einer südafrikanischen Kohorte haben gezeigt, dass weiße Kohorten ein höheres Risiko haben, an Myasthenia gravis zu erkranken, während schwarze Kohorten ein höheres Risiko für okuläre Manifestationen haben, die behandlungsresistent sind [207].

\section{Endokrinologie}

Die höchsten Prävalenzraten für Typ-1-Diabetes wurden in europäischen Ländern wie Finnland festgestellt, die niedrigsten Raten in südamerikanischen und asiatischen Ländern wie Venezuela und China [208]. Anderen Berichten zufolge weisen Amerika und Afrika die höchste bzw. niedrigste Prävalenz auf [209]. Darüber hinaus deutete eine groß angelegte Studie darauf hin, dass die genetische Prädisposition für Typ-1-Diabetes bei nichthispanischen weißen Personen am höchsten war [210].

Umweltfaktoren beeinflussen die Prävalenz des Morbus Basedow, da Länder mit Jodmangel eine höhere Prävalenz aufweisen [211]. Es gibt nur wenige Informationen über den Zusammenhang zwischen Morbus Basedow und ethnischer Zugehörigkeit. Im Jahr 2014 haben McLeod et al. erstmals Beweise dafür geliefert, dass Morbus Basedow bei der Bevölkerung der Schwarzen und der asiatisch-pazifischen Inselbewohner häufiger vorkommt [212]. Da es in Entwicklungsländern an umfassenden bevölkerungsbezogenen Studien mangelt, ist mehr Forschung erforderlich, um einen spezifischen Einblick in den Morbus Basedow zu erhalten, im Gegensatz zur Hyperthyreose im Allgemeinen.

Obwohl frühere Befunde darauf hindeuteten, dass die HashimotoThyreoiditis in der weißen Bevölkerung häufiger vorkommt [213], deuten neuere Untersuchungen darauf hin, dass japanische und koreanische Patienten mit Morbus Basedow ein höheres Risiko für Krankheitskomplikationen haben als kaukasische Menschen [214]. Darüber hinaus hat eine Studie in einer südafrikanischen Kohorte gezeigt, dass weiße Bevölkerungsgruppen ein höheres Risiko haben, an Myasthenia gravis zu erkranken. Schwarze Bevölkerungsgruppen haben ein höheres Risiko für okuläre Manifestationen, die behandlungsresistent sind [207].

Neuere Daten deuten darauf hin, dass Kaukasier ein mehr als 6-mal höheres Risiko haben, an GO zu erkranken [215], während GO bei asiatischen Patienten im Allgemeinen weniger schwerwiegend ist [215] und die Muskeln weniger betroffen sind. In asiatischen Bevölkerungsgruppen treten Komplikationen wie Katarakte häufiger auf [216]. 


\section{Dermatologie}

Psoriasis tritt in den westlichen Ländern häufiger auf [217], doch könnte der Mangel an Untersuchungen bei nichtkaukasischen Gruppen mit dieser Krankheit zu diesen Ergebnissen beitragen. In Amerika war die Prävalenz der Psoriasis in kaukasischen Gruppen fast doppelt so hoch wie in afroamerikanischen und hispanischen Gruppen [218]. Insbesondere wurde in einer Studie festgestellt, dass die Prävalenz der Psoriasis mit zunehmender Entfernung vom Äquator ansteigt, was auf die wichtige Rolle von Umweltfaktoren wie dem Sonnenlicht bei der Psoriasis hinweist. Yan et al. berichteten, dass farbige Menschen, die an Psoriasis leiden, eine geringere Lebensqualität und eine geringere Wahrscheinlichkeit einer Diagnose haben [219]. Wie aus Fallstudien hervorgeht, kann eine verzögerte Diagnose und Behandlung aufgrund der zugrunde liegenden okulären Manifestationen zu einem irreversiblen Sehverlust führen.

Die Prävalenz der systemischen Sklerose ist in Nordamerika und Australien höher als in Japan und Europa [181]. Darüber hinaus kann ein niedrigerer sozioökonomischer Status mit einem schwereren Krankheitsverlauf und einer Manifestation einer systemischen Sklerose bei afroamerikanischen Patienten in Verbindung gebracht werden [220], was den Einfluss von Umweltfaktoren auf die Schwere der Erkrankung bestätigt.

Medikamentenbedingte okuläre Nebenwirkung bei verschiedenen Autoimmunkrankheiten

Neben den Autoimmunkrankheiten können auch die zur Behandlung dieser Krankheiten eingesetzten Medikamente das Auge beeinträchtigen. Daher müssen Kliniker ein empfindliches Gleichgewicht zwischen einer wirksamen Behandlung und der Minimierung von Nebenwirkungen bei den Patienten wahren, indem sie auf die am meisten gefährdeten Patienten achten. Insgesamt sind die verschiedenen Therapien, die zur Behandlung von Autoimmunkrankheiten eingesetzt werden, sicher und wirksam. Die verschiedenen häufigen Nebenwirkungen häufig verwendeter Medikamente zur Behandlung von Autoimmunerkrankungen sind in Tabelle 3 aufgeführt.

\section{Fazit}

Obwohl sie oft unterschätzt und übersehen werden, treten bei allen in dieser Übersicht besprochenen Autoimmunkrankheiten zahlreiche Augenkomplikationen auf, die von leichten Sympto- men bis hin zu sehkraftbedrohenden Szenarien reichen. Mangelndes Krankheitsbewusstsein, Fehldiagnosen und nicht rechtzeitig eingeleitete Behandlungen können verheerende, dauerhafte Auswirkungen auf das Sehvermögen der Patienten haben. Insgesamt nimmt die weltweite Prävalenz von Autoimmunerkrankungen zu, wie epidemiologische Studien und Marktberichte zeigen. Es wird erwartet, dass dieser beobachtete Anstieg der Zahlen aufgrund von Umweltfaktoren, wie z.B. der Übernahme eines westlicheren Lebensstils in den östlichen Ländern, weiter anhalten wird. Bei vielen Autoimmunkrankheiten beeinflussen auch genetische Faktoren die Krankheitsveranlagung und den Schweregrad, wobei meistens die Frauen schwerer betroffen sind. Die Identifizierung der Personen mit dem höchsten Risiko für Autoimmunerkrankungen und den damit verbundenen okulären Manifestationen kann den Ärzten helfen, Diagnosen zu stellen, Screenings durchzuführen und Behandlungen effektiver umzusetzen, selbst bei asymptomatischen Patienten oder bei Patienten mit subtilen okulären Symptomen. Das Auge kann in vielen Fällen als Indikator für eine Grunderkrankung dienen. Daher sollten Kliniker dieses wichtige Instrument nutzen und keine geringfügigen Augensymptome übersehen, die in Abwesenheit offensichtlicher krankheitsspezifischer Symptome auftreten.

\section{Beiträge der Verfasser}

KG: Redaktion - erster Entwurf, Redaktion - Überprüfung und Bearbeitung. DM: Redaktion - Überprüfung und Bearbeitung. TRRS: Projektverwaltung, Ressourcen, Überwachung, Redaktion - Überprüfung und Bearbeitung. Alle Autoren haben an dem Artikel mitgewirkt und die vorgelegte Fassung genehmigt.

\section{Finanzierung}

Dieses Projekt wird durch das Forschungs- und Innovationsprogramm Horizont 2020 der Europäischen Union im Rahmen der Marie-SkłodowskaCurie-Maßnahmen (Finanzhilfevereinbarung - Nr. 813440) finanziert. DM wird durch das Horizon 2020 Orbital ITN Projekt finanziert.

\section{Lizenzangabe}

Glover K, Mishra D, Singh TRR. Epidemiology of Ocular Manifestations in Autoimmune Disease. Front Immunol. 2021;12:744396 (DOI: 10.3389/ fimmu.2021.744396). ${ }^{\circledR} 2021$ Glover, Mishra and Singh (Übersetzung), lizensiert unter CC BY 4.0 (https://creativecommons.org/licenses/by/4.0/ deed.de).

\section{Literatur}

Die Literatur ist unter www.karger.com/doi/10.1159/000521961 abrufbar. 